\title{
Effect of Renal Impairment on the Pharmacokinetics and Pharmacodynamics of Tofogliflozin (A SELECTIVE SGLT2 Inhibitor) in Patients with Type 2 Diabetes Mellitus
}

\author{
Authors \\ Sachiya Ikeda1, Yasuki Takano², Dietmar Schwab ${ }^{3}$, Agnes Portron ${ }^{3}$, Nahoko Kasahara-Ito ${ }^{4}$, Tomohisa Saito ${ }^{4}$, \\ Satofumi lida ${ }^{4}$
}

\section{Affiliations}

1 Primary lifecycle Management Department, Chugai Pharmaceutical Co., Ltd., Tokyo, Japan

2 Clinical Science and Strategy Department, Chugai Pharmaceutical Co., Ltd., Tokyo, Japan

3 Pharmaceutical Sciences, Clinical Pharmacology, Roche Pharma Research and Early Development, Roche Innovation Center Basel, Basel, Switzerland

4 Translational Clinical Research, Clinical Pharmacology Department, Chugai Pharmaceutical Co., Ltd., Tokyo, Japan

\section{Key words}

tofogliflozin, renal impairment, urinary glucose excretion, type 2 diabetes mellitus

received $\quad 12.04 .2018$

accepted 17.07.2018

\author{
Bibliography \\ DOI https://doi.org/10.1055/a-0662-0209 \\ Published online: 13.8 .2018 \\ Drug Res 2019; 69: 314-322 \\ (c) Georg Thieme Verlag KG Stuttgart · New York \\ ISSN 2194-9379
}

\section{Correspondence}

Sachiya Ikeda

Primary Lifecycle Management Department

Chugai Pharmaceutical Co.

Ltd. 2-1-1 Nihonbashi Muromachi

Chuo-ku

Tokyo 103-8324

Japan

Tel.: + 81/3/3273 4368, Fax: + 81/3/32810191

ikedascy@chugai-pharm.co.jp

\section{ABSTRACT}

Purpose Tofogliflozin is an orally available selective inhibitor of sodium-glucose co-transporter 2 for treatment of type 2 diabetes mellitus (T2DM). Two studies were conducted to evaluate the effect of renal impairment on pharmacokinetics and pharmacodynamics of tofogliflozin.

Methods The studies were: 1 ) single dose study in T2DM patients with normal renal function and mild, moderate and severe renal impairment, and 2) multiple dose study for 24 weeks in T2DM patients with normal renal function and moderate renal impairment.

Results Renal function did not have a clinically relevant effect on the PK of tofogliflozin. Urinary glucose excretion up to $24 \mathrm{~h}$ after administration of tofogliflozin (UGE24h) decreased with decreasing glomerular filtration rate. Lowering UGE24h resulted in waning glycemic control but not body weight reduction. Conclusions Single and multiple administrations of tofogliflozin were generally well tolerated in T2DM patients with various renal functions. As far as investigated here, these studies indicate no dose adjustment is required for patients with renal impairment.

\section{Introduction}

The World Health Organization reports that an estimated 422 million adults were living with diabetes worldwide in 2014 and that the global prevalence (age-standardized) of diabetes has nearly doubled since 1980 (from 4.7 to $8.5 \%$ ) in the adult population [1]. This is due to the increasing numbers of people with risk factors for diabetes, such as being overweight or obese. Diabetes is no longer a disease seen only in advanced wealthy countries; its prevalence is increasing all over the world. 
Sodium-glucose cotransporter-2 (SGLT2) inhibitors are the newest class of oral antidiabetic agents. The hypoglycemic effect of SGLT2 inhibitors is elicited by inhibiting the reabsorption of glucose through SGLT2 in the kidneys to increase urinary glucose excretion (UGE). In addition to the blood glucose lowering effect, there is accumulating evidence of comprehensive improvement of diabetes/ obesity-associated metabolic abnormalities due to the unique mode of action of SGLT2 inhibitors. Briefly, as a result of the inhibition of reabsorption of glucose and sodium ions in the proximal renal tubules, diuretic and hypotensive action and improvement of glomerular hyperfiltration are expected [2]. Increased UGE leads to a reduction in body weight and to changes in metabolism from glucose oxidation to lipid oxidation which contributes to an improvement of fatty liver disease and a reduction in visceral adipose tissue [3-6]. Treatment with SGLT2 inhibitors also results in reduced serum uric acid [7]. Tofogliflozin is a novel, highly selective SGLT2 inhibitor recently approved and launched in Japan for the treatment of type 2 diabetes mellitus (T2DM) [8-10]. Some of the basic pharmacokinetic characteristics of tofogliflozin have been reported. The in vitro study has suggested that tofogliflozin is metabolized to the carboxylated form (M1) and ketone form (M5) through hydroxylated derivatives (M4 and M2/3) by CYP2C18, $3 \mathrm{~A} 4 / 5,4 \mathrm{~A} 11$, and $4 \mathrm{~F} 3 \mathrm{~B}$, and tofogliflozin-derived substances are mainly eliminated by urinary excretion [11]. The carboxylated form is a major metabolite in humans and pharmacologically inactive. The ketone form is a minor metabolite in humans with weak pharmacological activity [11]. A human mass balance study combined with intravenous microdosing has demonstrated high oral bioavailability (BA) (97.5\%) of tofogliflozin [12]. Tofogliflozin is rapidly and completely absorbed and approximately $76 \%$ of total dose is excreted in urine and about $20 \%$ in feces within $72 \mathrm{~h}$ post-dose [13]. Urinary elimination of parent tofogliflozin however is a minor route of excretion, renal clearance accounting for only $16 \%$ of the parent drug systemic clearance, suggesting that dose adjustment of tofogliflozin in patients with renal impairment is unlikely needed as long as renal impairment does not affect extra-renal disposition of tofogliflozin. A moderate unbound fraction of 0.17 and presence of active tubular secretion of tofogliflozin ensures a high exposure of the luminal region of the kidney as the target organ despite the low contribution of renal clearance to total plasma clearance. Tofogliflozin exhibits dose-proportional and time-independent pharmacokinetics (data on file) and achieves readily steady-state plasma exposures due to an apparent terminal plasma half-life of about 6 days. Basic pharmacokinetics (PK) and pharmacodynamics (PD) profiles of tofogliflozin with healthy subjects are reported and tofogliflozin increases UGE as the dose increases [14]. Single PK profile of tofogliflozin with or without representative anti-T2DM drugs was evaluated in drug-drug interaction study and exposure of tofogliflozin was not affected by concomitant drugs and vice versa [15].

The exposure level of some oral antidiabetic agents is affected by renal function, and these agents should be prescribed carefully for such patients [16]. It is thus of great interest to understand the in-depth profiles of SGLT2 inhibitors in T2DM patients with chronic renal impairment. Here we report the PK/PD profiles of tofogliflozin treatment in T2DM patients who had either normal renal function or chronic renal impairment.

\section{Materials and Methods}

The following two clinical studies of tofogliflozin were conducted. (1) Single dose (SD) study: a study in T2DM patients with normal renal function and T2DM patients with mild, moderate and severe renal impairment. (2) Multiple dose (MD) study: an open study in T2DM patients with normal renal function and T2DM patients with moderate renal impairment over 24 weeks. All studies were conducted in accordance with the Declaration of Helsinki [17], the Good Clinical Practice, and the International Conference on Harmonization guidelines. The studies were approved by the Institutional Review Board of Rainier Clinical Research Center and Russian Peoples' Friendship University (for SD) and Tokyo Jikei University School of Medicine, Katsushika Medical Center (for MD). All patients gave written informed consent prior to participation. SD study was registered with ClinicalTrials.gov, number NCT00933972 and MD study was registered with the Japanese Pharmaceutical Information Center (registration number: JapicCTI-111572).

\section{Patients}

In the SD study, both male and female patients with T2DM and an estimated glomerular filtration rate (eGFR; calculated with MDRD formula [18]) of $>80 \mathrm{~mL} / \mathrm{min} / 1.73 \mathrm{~m}^{2}$ (normal renal function), $\geq 50$ and $\leq 80 \mathrm{~mL} / \mathrm{min} / 1.73 \mathrm{~m}^{2}$ (mild renal impairment), $\geq 30$ and $<50 \mathrm{~mL} / \mathrm{min} / 1.73 \mathrm{~m}^{2}$ (moderate renal impairment) or $<30 \mathrm{~mL}$ / $\mathrm{min} / 1.73 \mathrm{~m}^{2}$ (severe renal impairment) were eligible for inclusion. The eGRF cut-offs for the SD study were based on regulatory guidance at the time $[19,20]$, however during the study it was decided to include up to 4 additional patients with eGFR $90 \mathrm{~mL} / \mathrm{min} / 1.73 \mathrm{~m}^{2}$ as per regulatory recommendation to comply with the K/DOQI Clinical Practice Guideline for Chronic Kidney Disease from the National Kidney Foundation [21]. At screening, patients were required to be $\geq 40$ and $<80$ years of age, have a body mass index (BMI) of $\geq 18$ and $<40 \mathrm{~kg} / \mathrm{m}^{2}$, and have a glycated hemoglobin level (HbA1c; NGSP) of $\geq 6 \%$ and $<10.5 \%$. In addition, eligible patients had to have been treated with stable dose or regimen for renal impairment or diabetes (except SGLT2 inhibitor) at least 2 months before study drug administration. In the MD study, both male and female Japanese patients with T2DM and eGFR (calculated with the Japanese GFR estimation equation recommended by the Japanese Society of Nephrology [22]) of $\geq 90 \mathrm{~mL} / \mathrm{min} / 1.73 \mathrm{~m}^{2}$ (normal renal function) or $\geq 30$ and $<60 \mathrm{~mL} / \mathrm{min} / 1.73 \mathrm{~m}^{2}$ (moderate renal impairment) were eligible for inclusion in this study [21]. At screening, patients were required to be $\geq 20$ and $<75$ years of age, have a BMI of $\geq 18.5$ and $<45.0 \mathrm{~kg} / \mathrm{m} 2$, and have an HbA1c of $\geq 6.8$ and $<10.3 \%$. In addition, eligible patients had to have been treated with either diet and exercise alone or diet and exercise plus a stable dose of one oral hypoglycemic agent (except SGLT2 inhibitor) for $\geq 8$ weeks before screening. The concomitant therapies were kept during the study in principle. In both studies, patients were excluded if they were diagnosed as having type 1 diabetes or had had a complication and/or a history of diabetic ketoacidosis or end stage renal disease requiring dialysis, severe hypoglycemic episode, or repeated genitourinary tract infections within the 24 weeks before study drug administration (for SD) or screening (for MD). Other key exclusion criteria included complications and/or a history of malignant tumor, gastrointestinal disorder, respiratory 
disorder, hematological disorder, neurological disorder, psychiatric disorder, or immunological disorder.

\section{Study design}

SD study

This was a single-dose, non-randomized, open-label, parallel-group study. A group matching procedure was chosen to ensures that the 4 groups were comparable with respect to mean age (range of \pm 10 years), mean BMI (range of $\pm 20 \%$ ), and gender. Group matching started with the selection of 2 to 3 patients from the mild or moderate renal impairment group. Means for age and BMI of these patients were calculated as temporary means to be used to determine the means \pm given ranges for age and $\mathrm{BMI}$ for the next 2 to 3 patients to be included into the study in the other category. The proportion of men and women participating in each of the 4 groups was closely matched as much as possible aiming for recruitment of at least 3 patients of each gender in each of the 4 renal function groups. Patients with normal renal function were selected to match the patients in the other renal function groups. Patients from the severe renal impairment group were only enrolled once plasma PK, adverse events and clinical safety laboratory test data of at least 6 patients in the moderate renal impairment group were favorably assessed. Patients were orally administered a single dose of tofogliflozin ( $20 \mathrm{mg}$, capsule) in a fasted condition (at least $8 \mathrm{~h}$ ) on the morning of Day 1 . Blood samples for PK assessment were collected before administration and at 0.5, 1, 1.5, 2, 3, 4, 6, 8, 10, 12, $16,24,36$, and $48 \mathrm{~h}$ after administration. Urine samples for PK and PD assessment were collected for $48 \mathrm{~h}$ after administration.

\section{MD study}

This was a randomized, open-label, parallel-group study. Patients were administered tofogliflozin ( $40 \mathrm{mg}$, tablet) once daily at $15 \mathrm{~min}$ before breakfast for 24 weeks. Blood samples for trough concentration assessment were collected before administration and at Week 4, 8, 12, 16, 20 and 24 from all patients. Patients who consented to full PK sampling protocol were subjected to frequent sampling; before and at $0.5,1,1.5,2,3,4,6,8,10,12,16$ and $24 \mathrm{~h}$ after the first administration. Urine samples for PK and PD assessment were collected from $24 \mathrm{~h}$ before to $24 \mathrm{~h}$ after the first administration by the full PK sampling protocol. HbA1c and fasting plasma glucose (FPG) changes as a glycemic parameter and body weight change were monitored during the study.

\section{PK analysis}

In the SD study, the plasma and urine concentrations of tofogliflozin and its metabolites were measured in F. Hoffman-La Roche Ltd. (Basel, Switzerland). The lower limit to upper limit of quantification range for tofogliflozin and the carboxylate metabolite were the same and were $0.2-500 \mathrm{ng} / \mathrm{mL}$ and $5-1000 \mathrm{ng} / \mathrm{mL}$ for plasma and urine, respectively. The plasma and urine quantification ranges for the ketone form were $0.5-500 \mathrm{ng} / \mathrm{mL}$ and $10-1000 \mathrm{ng} / \mathrm{mL}$ for plasma and urine, respectively. In the MD study, the plasma and urine concentrations of tofogliflozin and its metabolites were measured in Swiss BioAnalytics AG (Birsfelden, Switzerland). The plasma and urine quantification range for tofogliflozin were $0.200-500 \mathrm{ng} / \mathrm{mL}$ and $10.0-10000 \mathrm{ng} / \mathrm{mL}$, respectively. The quantification ranges for the carboxylated and ketone were the same and were $0.500-$ $500 \mathrm{ng} / \mathrm{mL}$ and $10.0-10000 \mathrm{ng} / \mathrm{mL}$ for plasma and urine, respectively.

For all analytes, the PK parameters of $C_{\max }$ (maximum plasma drug concentration), $T_{\max }$ (time to reach $C_{\max }$ ), $t_{1 / 2}$ (elimination half-life), $A U C_{\text {inf }}$ (area under the plasma concentration-time curve from time zero to infinity), CL/F (apparent oral clearance), Vd/F (apparent volume of distribution) and $\mathrm{CL}_{\mathrm{r}}$ (renal clearance) were determined by non-compartmental analysis using WinNonlin ver. 6.1 software (Pharsight Corporation, Mountain View, CA, USA).

\section{PD analysis}

In both studies, the PD profile of tofogliflozin was evaluated by UGE24h, i. e., cumulative urinary glucose excretion $24 \mathrm{~h}$ after administration. Renal glucose reabsorption inhibition was calculated as the ratio of renal glucose clearance over eGFR at same time interval with SD study data.

\section{Safety assessments}

Safety was evaluated via adverse events (AEs), clinical laboratory tests, vital signs, and standard 12-lead electrocardiogram (ECG) examinations. Fluid balance and glucose monitoring were also performed as additional safety tests.

\section{Results}

\section{Demographics of the subjects}

In the SD study, 36 patients were considered eligible, and all patients were administered the study drug: 11 patients with normal renal function, 8 patients with mild renal impairment, 9 patients with moderate renal impairment and 8 patients with severe renal impairment. 34 out of 36 patients were treated with concomitant antidiabetic medications including biguanides $(N=17)$, sulfonylureas $(N=16)$, thiazolidinediones $(N=3), G L P-1$ agonist $(N=1)$ and insulins ( $N=18)$. In the MD study, 13 patients were randomized in the normal renal function group and 30 patients were randomized in the moderate renal impairment group. Of the 15 patients who enrolled in the full PK sampling protocol, 8 had normal renal function and 7 had moderate renal impairment. 20 out of 43 patients were treated with concomitant antidiabetic medications including sulfonylureas ( $N=15)$, and DPP4 inhibitors $(N=5)$. All patients completed the study and their demographics and baseline characteristics are shown in $>$ Table $\mathbf{1}$.

\section{PK profile}

In the SD study, plasma and urinary concentrations of tofogiflozin and 2 metabolites were measured to investigate the effect of renal impairment on the PK profiles of tofogliflozin and its metabolites. After rapid absorption, tofogliflozin concentrations peaked $1 \mathrm{~h}$ after administration, and subsequently, tofogliflozin plasma concentration declined in a biphasic manner indicative of a rapid distribution phase and a slower elimination phase ( $>$ Fig. 1a). The tofogliflozin plasma concentration time profile was similar regardless of the renal function status. Mean $\mathrm{AUC}_{\text {inf }}$ was about $19 \%, 17 \%$ and $13 \%$ higher for patients with mild, moderate and severe renal impairment, respectively, as compared to patients with normal renal function. 
- Table 1 Demographic and baseline characteristics.

\begin{tabular}{|c|c|c|c|c|c|c|c|c|}
\hline \multirow[t]{2}{*}{ Study } & \multirow[t]{2}{*}{$\begin{array}{l}\text { Renal } \\
\text { Function }\end{array}$} & \multirow[t]{2}{*}{$N$} & Age & Sex $^{a}$ & $\begin{array}{c}\text { Body } \\
\text { weight }\end{array}$ & BMI & HbA1c & eGFR \\
\hline & & & (years) & $\begin{array}{c}\text { Male/ } \\
\text { Female }\end{array}$ & (kg) & $\left(\mathrm{kg} / \mathrm{m}^{2}\right)$ & (\%) & $\left(\mathrm{mL} / \mathrm{min} / 1.73 \mathrm{~m}^{2}\right)$ \\
\hline \multirow[t]{4}{*}{ SD } & Normal & 11 & $63.4(5.3)$ & $5 / 6$ & $94.1(13.4)$ & $33.77(4.048)$ & $7.57(1.20)$ & $102.3(17.6)$ \\
\hline & Mild & 8 & $67.3(4.1)$ & $4 / 4$ & $90.8(11.0)$ & $32.90(3.598)$ & $6.79(0.536)$ & $66.4(8.1)$ \\
\hline & Moderate & 9 & $63.4(5.8)$ & $4 / 5$ & $89.3(11.9)$ & $33.31(2.676)$ & $7.51(1.04)$ & $38.6(5.1)$ \\
\hline & Severe & 8 & $63.5(3.8)$ & $5 / 3$ & $91.0(12.5)$ & $33.16(3.857)$ & $7.00(0.661)$ & $18.5(6.4)$ \\
\hline \multirow[t]{2}{*}{ MD } & Normal & 13 & $55.3(12.4)$ & $11 / 2$ & $69.1(12.4)$ & $24.94(3.030)$ & $7.89(0.755)$ & $105.47(15.042)$ \\
\hline & Moderate & 30 & $64.2(7.2)$ & $24 / 6$ & $69.9(9.9)$ & $26.05(3.406)$ & $7.33(0.98)$ & $49.25(11.843)$ \\
\hline
\end{tabular}
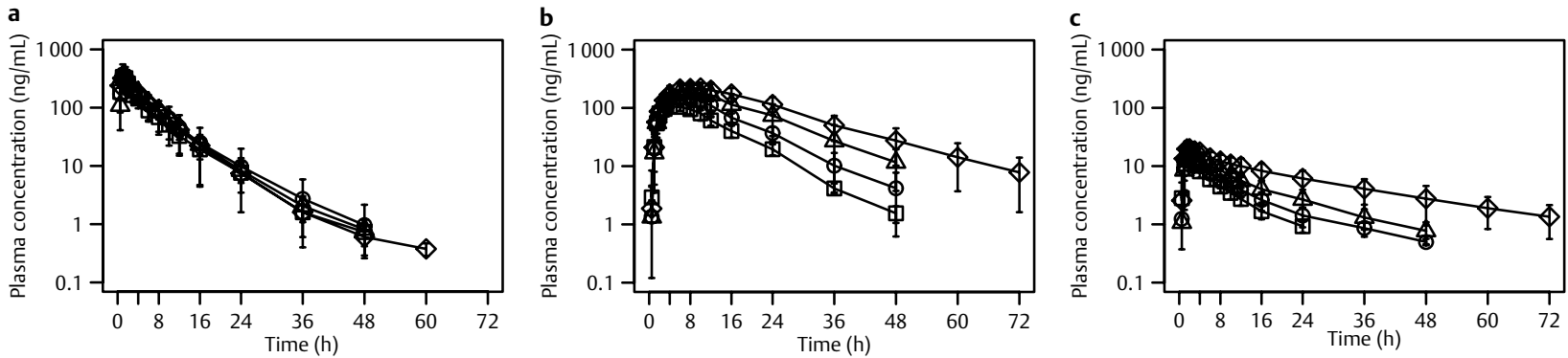

- Fig. 1 Plasma concentration profile (mean \pm SD) of a tofogliflozin, $\mathbf{b}$ carboxylated form and $\mathbf{c}$ ketone form after single administration to T2DM patients with normal renal function $(\square)$, mild $(\circ)$, moderate $(\Delta)$ and severe renal impairment $(\diamond)$.

The plasma concentration of the carboxylated form peaked at approximately $6 \mathrm{~h}$ after administration of tofogliflozin and decreased slowly ( $\mathbf{F i g}$. 1b). The plasma concentration-time profile of the ketone form peaked $1.5 \mathrm{~h}$ after administration in patients with normal renal function and was parallel to the parent drug ( $\triangleright$ Fig. 1c). Unlike the parent drug, the elimination of the two metabolites was highly affected by the renal function status, hence the mean exposure of the carboxylated form and ketone form drastically increased with declining renal function. Mean $A U C_{\text {inf }}$ of thecarboxylated form in patients with moderate renal impairment and the ketone form in patients with severe renal impairment was up to 3.6- and 4.1-fold of that in patients with normal renal function, respectively. Renal clearance of all 3 measured analytes distinctly decreased with declining renal function. In the PK assessment of the MD study utilizing the full PK sampling protocol, the change in the PK profile as renal function changed was similar to that observed in the SD study, even though a higher dose was used. PK parameters of tofogliflozin, carboxylated form and ketone form are summarized in > Table 2-4, respectively. The plasma trough concentration of tofogliflozin in patients with normal renal function and patients with moderate renal impairment at week 24 were 29.8 (18.7) and 41.8 (50.3) ng/mL, respectively [mean (standard deviation; SD)]. The plasma trough concentration of the carboxylated and ketone forms of tofogliflozin were 54.5 (39.7) and 119 (71.9) $\mathrm{ng} / \mathrm{mL}$, respectively in patients with normal renal function and 2.37 (1.51) and 8.37 (6.19) ng/mL, respectively in patients with moderate renal impairment [mean $(\mathrm{SD})]$.

\section{PD profile}

UGE24h was analyzed for the SD study and part of the MD study, where the full PK sampling protocol was applied. UGE24h is summarized in $>$ Table 5. UGE24h decreased with declining renal function. The UGE24h after single administration of tofogliflozin for patients with normal renal function and patients with mild, moderate and severe renal impairment were 81.5 (34.0), 47.2 (29.9) 21.2 (8.86) and 11.9 (7.27) g, respectively [mean (SD)]. Scatterplot of UGE24h change from pre-dose versus eGFR, using data from both the SD and the MD studies, revealed higher UGE24h change observed in patients with higher eGRF ( $\triangleright$ Fig. 2), while no significant correlation was found between the inhibition of renal glucose reabsorption and eGFR in the SD study ( $>$ Fig. 3). Values of percentage inhibition of renal glucose reabsorption in patients with normal renal function and patients with mild, moderate and severe renal impairment were 32.0 (11.0), 32.6 (15.1), 27.4 (11.6), 36.7 (13.4) \%, respectively [mean (SD)].

\section{Safety}

In the SD study, 3 AEs were reported by 6 patients with normal renal function and $4 \mathrm{AEs}$ by 3 patients with moderate renal impairment. There were no serious AEs reported during the study. There was no apparent pattern of AEs according to severity of renal impairment. All AEs were of mild intensity except one case of hypoglycemia (of moderate intensity) in a patient with moderate renal impairment. Abnormal laboratory parameters were reported during the study, but no obvious correlation according to severity of renal impair- 


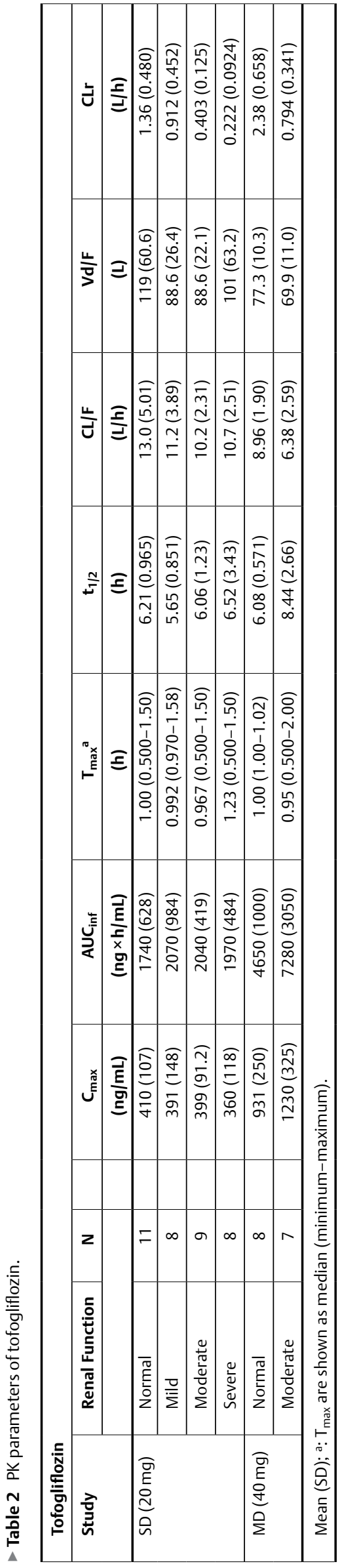

ment, and mean changes from baseline were minimal. In the MD study, 15AEs in 8 patients with normal renal function and $24 \mathrm{AEs}$ in 16 patients with moderate renal impairment were reported. Of these, $7 \mathrm{AEs}$ in 3 patients in the normal renal function group and 5 AEs in 4 patients in the moderate renal impairment group were considered as having an undeniable causal relationship with the study drug. Details are shown in $>$ Table 6 . There were no deaths. One serious AE related to the study drug (rectal cancer in the normal renal function group) was reported, and it was the only case leading to discontinuation. The severity of symptoms was mild in all cases with the exception of the rectal cancer. No AEs with a clear imbalance of incidence attributable to the difference in renal function were detected. Laboratory data with abnormal changes identified in the normal renal function group were decreased neutrophil count, increased blood triglycerides, and increased WBC count in urine, and the incidence was $7.7 \%$ (1 of 13 patients) in each case. Laboratory data with abnormal changes identified in the moderate renal impairment group were decreased neutrophil count, increased blood urea nitrogen (BUN), increased blood triglycerides, increased creatinine phosphokinase (CPK), increased occult protein in urine, and increased of urine WBC count in urine, and the incidence was $3.3 \%$ ( 1 of 30 patients) in each case except for $10.0 \%$ ( 3 of 30 patients) of increased occult blood in urine.

Regarding renal function, although serum creatinine was slightly increased in both the normal renal function group and the moderate renal impairment group, eGFR was slightly decreased in the normal renal function group whereas eGFR was unchanged in the moderate renal impairment group at 24 week compared to baseline. Cystatin C was slightly increased in the moderate renal impairment group whereas no change in the normal renal function group and $\mathrm{N}$-acetyl $\beta$-D-glucosaminidase (NAG) was slightly decreased in both groups at 24 week from baseline. $\beta 2$-microglobulin was no obvious change in both groups during the study.

\section{Glycemic parameters and body weight change}

Values of HbA1c, FPG and body weight change from baseline to Week 24 were -0.65 (0.91) \%, -29.8 (32.0) mg/dL and -2.20 (3.46) $\mathrm{kg}$, respectively in patients with normal renal function $(\mathrm{N}=11)$ and $-0.23(0.67) \%,-16.7(22.3) \mathrm{mg} / \mathrm{dL}$ and $-1.75(2.00) \mathrm{kg}$, respectively in patients with moderate renal impairment $(\mathrm{N}=29)$ [mean (SD)]. Time course of HbA1c, FPG and body weight change from baseline to Week 24 are shown ( $\vee$ Fig. $4 a-c)$. Control of glycemic parameters by tofogliflozin treatment was attenuated as renal function declined. However, body weight decrease was not markedly affected by the status of renal function.

\section{Discussion}

These studies were conducted to understand the effect of different degrees of renal impairment on the pharmacokinetics, pharmacodynamics and safety of single and multiple doses of tofogliflozin.

Previous studies reveal that tofogliflozin is rapidly and completely absorbed and approximately $76 \%$ of the total dose was excreted in urine and about $20 \%$ was found in feces within $72 \mathrm{~h}$ post-dose [13]. The general PK profile of tofogliflozin was also reported by Kasahara-Ito et.al. concluding that once daily treatment of tofogliflozin did not cause accumulation in plasma with time [14]. 
D Table 3 PK parameters of carboxylated form of tofogliflozin.

\begin{tabular}{|c|c|c|c|c|c|c|c|}
\hline \multicolumn{8}{|c|}{ Carboxylated form } \\
\hline \multirow[t]{2}{*}{ Study } & \multirow[t]{2}{*}{ Renal Function } & \multirow[t]{2}{*}{$\mathbf{N}$} & $C_{\max }$ & $A U C_{\text {inf }}$ & $T_{\max }{ }^{a}$ & $t_{1 / 2}$ & $\mathrm{CLr}$ \\
\hline & & & (ng/mL) & $(\mathrm{ng} \times \mathrm{h} / \mathrm{mL})$ & (h) & (h) & (L/h) \\
\hline \multirow[t]{4}{*}{ SD (20 mg) } & Normal & 11 & $112(24.3)$ & $1610(298)$ & $6.00(3.00-10.0)$ & $6.56(0.544)$ & $4.26(1.01)$ \\
\hline & Mild & 8 & $159(33.7)$ & $2590(434)$ & $6.03(4.00-8.17)$ & $7.23(1.06)$ & $2.76(0.563)$ \\
\hline & Moderate & 9 & $198(90.8)$ & $4070(2120)$ & $6.03(4.00-12.0)$ & $9.37(0.919)$ & $1.73(6.36)$ \\
\hline & Severe & 8 & $230(56.3)$ & $5850(1750)$ & $10.0(6.00-12.0)$ & $11.8(2.10)$ & $0.774(0.285)$ \\
\hline \multirow[t]{2}{*}{ MD (40 mg) } & Normal & 8 & $243(64.9)$ & $3450(615)$ & $4.00(3.00-6.03)$ & $8.23(1.21)$ & $4.32(0.864)$ \\
\hline & Moderate & 7 & 375 (104) & $9270(3620)$ & $6.00(5.92-6.00)$ & 15.7 (5.75) & $1.85(0.737)$ \\
\hline
\end{tabular}

- Table 4 PK parameters of ketone form of tofogliflozin.

\begin{tabular}{|c|c|c|c|c|c|c|c|}
\hline \multicolumn{8}{|l|}{ ketone form } \\
\hline \multirow[t]{2}{*}{ Study } & \multirow[t]{2}{*}{ Renal Function } & \multirow[t]{2}{*}{$\mathbf{N}$} & $C_{\max }$ & $\mathrm{AUC}_{\text {inf }}$ & $T_{\max }{ }^{a}$ & $t_{1 / 2}$ & $\mathrm{CLr}$ \\
\hline & & & (ng/mL) & $(\mathrm{ng} \times \mathrm{h} / \mathrm{mL})$ & (h) & (h) & $(\mathrm{L} / \mathrm{h})$ \\
\hline \multirow[t]{4}{*}{ SD (20 mg) } & Normal & 11 & $17.3(4.42)$ & $108(21.9)$ & $1.50(0.98-2.00)$ & $7.14(1.18)$ & $9.84(2.42)$ \\
\hline & Mild & 8 & $15.6(4.07)$ & $141(34.4)$ & $1.50(1.50-4.08)$ & $9.45(2.58)$ & $6.48(1.82)$ \\
\hline & Moderate & 9 & $17.7(5.30)$ & $211(57.1)$ & $1.50(0.98-3.05)$ & $13.4(2.00)$ & $3.81(1.50)$ \\
\hline & Severe & 8 & $21.5(5.74)$ & $440(173)$ & $2.00(0.97-4.00)$ & $19.5(4.05)$ & $1.42(0.636)$ \\
\hline \multirow[t]{2}{*}{ MD (40 mg) } & Normal & 8 & $28.4(7.56)$ & $206(35.1)$ & $1.50(1.00-2.00)$ & $7.67(0.847)$ & $11.9(2.56)$ \\
\hline & Moderate & 7 & $38.9(10.3)$ & $575(324)$ & $\begin{array}{c}1.50(0.967- \\
2.00)\end{array}$ & $14.4(6.92)$ & $4.22(1.76)$ \\
\hline
\end{tabular}

- Table 5 Cumulative UGE up to 24h before/after tofogliflozin administration.

\begin{tabular}{|l|l|l|c|c|c|}
\hline Study & Renal Function & $\mathbf{N}$ & UGE Pre-dose (g) & UGE Post-dose (g) & UGE Change from Pre-dose (g) \\
\hline \multirow{3}{*}{ SD (20 mg) } & Normal & 11 & $6.71(8.77)$ & $81.5(34.0)$ & $74.9(31.0)$ \\
\cline { 2 - 6 } & Mild & 8 & $8.80(17.0)$ & $47.2(29.9)$ & $38.4(17.3)$ \\
\cline { 2 - 6 } & Moderate & 9 & $2.00(3.76)$ & $21.2(8.86)$ & $19.2(6.79)$ \\
\cline { 2 - 6 } & Severe & 8 & $0.553(0.247)$ & $11.9(7.27)$ & $11.3(7.30)$ \\
\hline \multirow{2}{*}{ MD (40 mg) } & Normal & 8 & $38.6(40.4)$ & $138(41.7)$ & $99.7(28.9)$ \\
\cline { 2 - 6 } & Moderate & 7 & $2.46(3.17)$ & $47.0(14.5)$ & $44.6(15.4)$ \\
\hline Mean (SD) & \multicolumn{5}{|l}{} \\
\hline
\end{tabular}

The SD study demonstrated that renal impairment did not have a clinically significant effect on the pharmacokinetics of tofogliflozin. The lack of effect of renal impairment on tofogliflozin pharmacokinetics is consistent with urinary elimination being a minor route of excretion for tofogliflozin, with renal clearance accounting for only $16 \%$ of the parent drug systemic clearance [12]. As expected, renal clearance of tofogliflozin was clearly affected by renal impairment. Mean $A_{U} C_{\text {inf }}$ for both carboxylated form and ketone form increased with decreasing renal function (3.6 times for carboxylted form, 4.1 times for ketone form in the severe renal impairment group compared to the normal renal function group). Therefore, renal clearance is an important route for the elimination of the metabolites and impaired renal function results in increased exposure to those metabolites and increased metabolite to parent ratio. Following once daily treatment for 24 weeks, plasma trough concentrations of tofogliflozin and its metabolites did not change with time both in patients with normal renal function and in patients with moderate renal impairment. PK profiles of tofogliflozin were not affected to a clinically relevant degree by renal impairment. The higher systemic exposure of the carboxylated form in patients with renal impairment is not clinically relevant as it is an inactive metabolite. Furthermore, while the ketone form had also a higher exposure in patients with renal impairment as compared to patients with normal renal function, it is a minor metabolite in humans. Therefore, no dose adjustment is required for the treatment of patients with impaired renal function as far as investigated in these studies.

A pronounced UGE24h was observed after a single oral 20 or $40 \mathrm{mg}$ dose of tofogliflozin across all renal function categories as compared to baseline. UGE24h decreased with increasing renal impairment. This is mainly explained by a reduction of filtered glucose in the glomeruli due to decreased GFR as renal function decreased. 


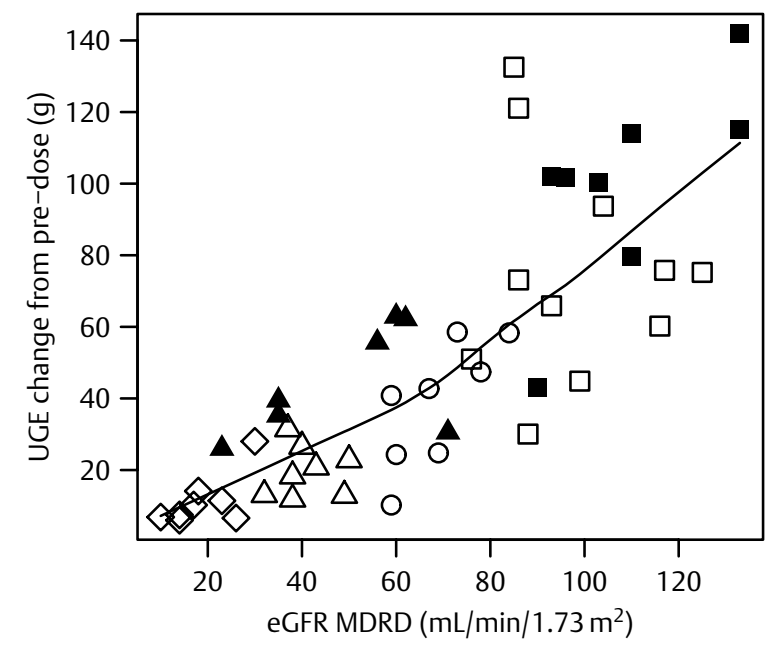

- Fig. 2 Relationship between UGE change from pre-dose and eGFR in T2DM patients with normal renal function $(\square)$, mild $(O)$, moderate $(\Delta)$ and severe renal impairment $(\diamond)$ in the SD study, and normal renal function $(\boldsymbol{\nabla})$ and moderate renal impairment $(\mathbf{\Delta})$ in the MD study. Black straight line indicates the smooth curve. Note: The definition of the categories of renal function differs between SD and MD study [eGFR $>80 \mathrm{~mL} / \mathrm{min} / 1.73 \mathrm{~m}^{2}$ (normal renal function), $\geq 50$ and $\leq 80 \mathrm{~mL} / \mathrm{min} / 1.73 \mathrm{~m}^{2}$ (mild renal impairment), $\geq 30$ and $<50 \mathrm{~mL} / \mathrm{min} / 1.73 \mathrm{~m}^{2}$ (moderate renal impairment) or $<30 \mathrm{~mL}$ / $\mathrm{min} / 1.73 \mathrm{~m}^{2}$ (severe renal impairment) in SD study and eGFR $\geq 90 \mathrm{~mL} / \mathrm{min} / 1.73 \mathrm{~m}^{2}$ (normal renal function) or $\geq 30$ and $<60 \mathrm{~mL} / \mathrm{min} / 1.73 \mathrm{~m}^{2}$ (moderate renal impairment) in MD study].

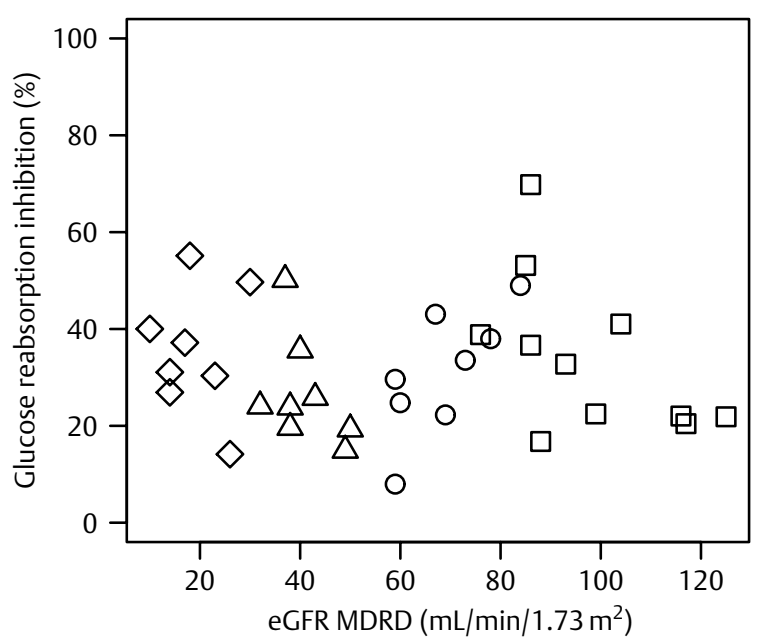

- Fig. 3 Relationship between glucose reabsorption inhibition and eGFR in T2DM patients with normal renal function $(\square)$, mild $(\odot)$, moderate $(\Delta)$ and severe renal impairment $(\diamond)$ in the SD study.

Interestingly, the inhibition of renal glucose reabsorption had no clear correlation with eGFR and the percentage of inhibition tend to be constant regardless of the degree of renal impairment. UGE24h observed in the SD study seems to be slightly lower than that observed in the MD study. This might be due to somewhat
- Table 6 Summary of safety profiles in MD study.

\begin{tabular}{|c|c|c|}
\hline & $\begin{array}{l}\text { Normal renal } \\
\text { function } n=13\end{array}$ & $\begin{array}{l}\text { Moderate renal } \\
\text { impairment } \\
n=30\end{array}$ \\
\hline Patients with at least one $\mathrm{AE}(\%)$ & $8(61.5)$ & $16(53.3)$ \\
\hline Patients with drug-related SAEs (\%) & $1(7.7)$ & $0(0)$ \\
\hline $\begin{array}{l}\text { Patients with SAEs leading to } \\
\text { discontinuation (\%) }\end{array}$ & $1(7.7)$ & $0(0)$ \\
\hline Any AE & 15 & 24 \\
\hline Nasopharyngitis & 3 & 5 \\
\hline Gastroenteritis & 1 & 1 \\
\hline Bronchitis & 1 & \\
\hline Influenza & 1 & \\
\hline Upper respiratory tract infection & 2 & \\
\hline Urinary tract infection & 1 & \\
\hline Skin infection & & 1 \\
\hline Chronic sinusitis & & 1 \\
\hline Musculoskeletal pain & & 1 \\
\hline Osteoarthritis & & 2 \\
\hline Headache & 1 & 1 \\
\hline Pollakiuria & & 3 \\
\hline Hyperlipidemia & & 1 \\
\hline Hypoglycemia & 1 & 1 \\
\hline Xeroderma & & 1 \\
\hline Seborrhoeic dermatitis & & 2 \\
\hline Abdominal discomfort & 1 & \\
\hline Constipation & 1 & \\
\hline Thirst & & 1 \\
\hline Diabetic retinopathy & & 2 \\
\hline Rectal cancer & 1 & \\
\hline Blood ketone body increased & 1 & \\
\hline Blood pressure decreased & & 1 \\
\hline
\end{tabular}

more severe diabetic condition with higher HbA1c in patients with normal renal function in the MD study compared to the SD study. Furthermore, the adopted dose of tofogliflozin was $40 \mathrm{mg}$ in the MD study, although a tofogliflozin dose of $20 \mathrm{mg}$ is close to the maximum effect for UGE induction [14].

Multiple administration of tofogliflozin for 24 weeks was equally well-tolerated in patients with normal renal function and with moderate renal impairment. Regarding glycemic parameter, $\mathrm{HbA} 1 \mathrm{c}$ and FPG changes from baseline were attenuated in patients with moderate renal impairment compared to those in patients with normal renal function. However, body weight reduction was almost the same between the two groups. This incongruity is also observed in other SGLT2 inhibitors and the reason remains unclear [23-27]. The inhibition of renal glucose reabsorption was constant across all renal function groups. This indicates that tofogliflozin reached its target in the kidney independent of renal function. Body weight reduction might be regulated in some way by sensing the renal glucose reabsorption inhibition.

It was recently reported that empagliflozin significantly lowered the risk of progression of kidney disease, as defined by incidence of worsening nephropathy, in patients with T2DM at high risk of cardiovascular events. That analysis was conducted according to the 

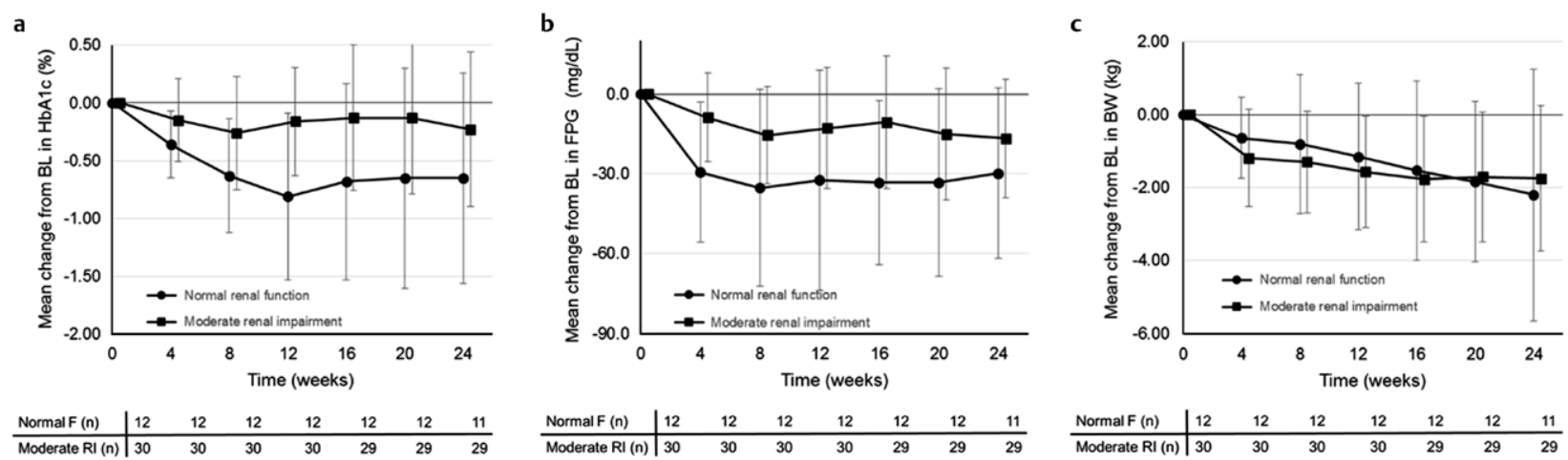

- Fig. 4 a Changes in HbA1c from baseline during the treatment period. $\mathbf{b}$ Changes in FPG from baseline during the treatment period. $\mathbf{c}$ Changes in body weight from baseline during the treatment period.

pre-specified analysis plan for one of the secondary outcomes of the EMP-REG OUTCOME trial [28]. In addition, the CANVAS study showed canagliflozin reduced cardiovascular and renal outcomes both in T2DM patients with prior cardiovascular events and without prior cardiovascular events [29]. One of putative mechanisms underlying the renoprotective effect would be driven by hemodynamic changes. SGLT2 inhibitors reduce sodium reabsorption in the proximal tubules, thereby increasing sodium delivery to the distal tubules where it leads to modulation of afferent arteriole resistance in the glomeruli through the macula densa and decreased filtration rate. The prognostic significance of diabetic hyperfiltration has so far been controversial, but a recent cohort study shows hyperfiltration contributes to renal function loss and to the onset or progression of nephropathy [30,31]. Further data accumulation will be needed, including that of other SGLT2 inhibitors, to conclude whether SGLT2 inhibitors have a renoprotective effect. In a clinical setting, when deciding on a prescription of SGLT2 inhibitor the prescriber should take into consideration his/her age, disease condition, complication and lifestyle and assess the benefit-risk balance for the patients.

\section{Conclusions}

The PK profiles of tofogliflozin were not affected to a clinically relevant degree by declining renal function. Although systemic exposure of the metabolites measured in the study was obviously increased with increasing renal impairment, their contribution to the efficacy was considered low. Administration of tofogliflozin at single or multiple doses for 24 weeks was safe and well tolerated in male and female patients with T2DM and varying degrees of renal function. Therefore, as far as investigated in these studies, no dose adjustment is required for patients with renal impairment. UGE24h decreased with decreasing renal function which resulted in waning glycemic control but no change in body weight reduction.

\section{Author contributions}

All authors meet the criteria for authorship as recommended by the International Committee of Medical Journal Editors (ICMJE). D.S. designed the SD study, and D.S and A.P. contributed to data acquisition of the SD study, and critically revised the draft manuscript. S.lkeda, Y.T., N.K., T.S., S.lida analyzed all the data in the manuscript and wrote the draft manuscript. All authors approved the final version to be published.

\section{Acknowledgments}

The authors thank all patients and staff for cooperating in the 2 studies sponsored by Roche Pharma Research and Early Development and Chugai Pharmaceutical Co., Ltd.

\section{Conflict of Interests}

Y.T., N.K., T.S. and S.lida and S.Ikeda are employees of Chugai Pharmaceutical Co., Ltd. D.S., and A.P. are employees of H. Hoffmann La Roche Co., Ltd.

\section{References}

[1] World Health Organization. Global report on diabetes. Geneva: World Health Organization; 2016

[2] De Nicola L, Gabbai FB, Liberti ME et al. Sodium/glucose cotransporter 2 inhibitors and prevention of diabetic nephropathy: Targeting the renal tubule in diabetes. Am J Kidney Dis 2014; 64: 16-24

[3] Obata A, Kubota N, Kubota T et al. Tofogliflozin improves insulin resistance in skeletal muscle and accelerates lipolysis in adipose tissue in male mice. Endocrinology. 2016; 157: 1029-1042

[4] Bolinder J, Ljunggren Ö, Johansson L et al. Dapagliflozin maintains glycaemic control while reducing weight and body fat mass over 2 years in patients with type 2 diabetes mellitus inadequately controlled on metformin. Diabetes Obes Metab 2014; 16: 159-169

[5] Blonde L, Stenlöf K, Fung A et al. Effects of canagliflozin on body weight and body composition in patients with type 2 diabetes over 104 weeks. Postgrad Med. 2016; 128: 371-380

[6] Ohki T, Isogawa A, Toda $\mathrm{N}$ et al. Effectiveness of ipragliflozin, a sodiumglucose co-transporter 2 inhibitor, as a second-line treatment for non-alcoholic fatty liver disease patients with type 2 diabetes mellitus who do not respond to incretin-based therapies including glucagonlike peptide- 1 analogs and dipeptidyl peptidase- 4 inhibitors. Clin Drug Investig 2016; 36: 313-319 
[7] Davies M], Trujillo A, Vijapurkar U et al. Effect of canagliflozin on serum uric acid in patients with type 2 diabetes mellitus. Diabetes Obes Metab. 2015; 17: 426-429 Erratum in: Diabetes Obes Metab. 2015; $17: 708$

[8] Suzuki M, Honda K, Fukazawa M et al. Tofogliflozin, a potent and highly specific sodium/glucose cotransporter 2 inhibitor, improves glycemic control in diabetic rats and mice. J Pharmacol Exp Ther 2012; 341: 692-701

[9] Kaku K, Watada H, Iwamoto $\mathrm{Y}$ et al. Efficacy and safety of monotherapy with the novel sodium/glucose cotransporter-2 inhibitor tofogliflozin in Japanese patients with type 2 diabetes mellitus: a combined Phase 2 and 3 randomized, placebo-controlled, double-blind, parallel-group comparative study. Cardiovasc Diabetol. 2014; 13: 65

[10] Tanizawa Y, Kaku K, Araki E et al. Tofogliflozin 004 and 005 Study group. Long-term safety and efficacy of tofogliflozin, a selective inhibitor of sodium-glucose cotransporter 2 , as monotherapy or in combination with other oral antidiabetic agents in Japanese patients with type 2 diabetes mellitus: Multicenter, open-label, randomized controlled trials. Expert Opin Pharmacother 2014; 15: 749-766

[11] Yamane M, Kawashima K, Yamaguchi K et al. In vitro profiling of the metabolism and drug-drug interaction of tofogliflozin, a potent and highly specific sodium-glucose co-transporter 2 inhibitor, using human liver microsomes, human hepatocytes, and recombinant human CYP. Xenobiotica 2015; 45: 230-238

[12] Schwab D, Portron A, Backholer Z et al. A novel double-tracer technique to characterize absorption, distribution, metabolism and excretion (ADME) of $\left[{ }^{14} \mathrm{C}\right]$ tofogliflozin after oral administration and concomitant intravenous microdose administration of $\left[{ }^{13} \mathrm{C}\right]$ tofogliflozin in humans. Clin Pharmacokinet 2013; 52: 463-473

[13] Zell M, Husser C, Kuhlmann O et al. Metabolism and mass balance of SGLT2 inhibitor tofogliflozin following oral administration to humans. Xenobiotica 2014; 44: 369-378

[14] Kasahara-Ito N, Fukase H, Ogama Y et al. Pharmacokinetics and pharmacodynamics of tofogliflozin (a selective SGLT2 inhibitor) in healthy male subjects. Drug Res (Stuttg) 2017; 67: 349-357

[15] Kasahara N, Fukase H, Ohba Y et al. A pharmacokinetic/pharmacodynamic drug-drug interaction study of tofogliflozin (a new SGLT2 inhibitor) and selected anti-type 2 diabetes mellitus drugs. Drug Res (Stuttg) 2016; 66: 74-81

[16] Betônico CC, Titan SM, Correa-Giannella ML et al. Management of diabetes mellitus in individuals with chronic kidney disease: Therapeutic perspectives and glycemic control. Clinics (Sao Paulo) 2016; 71: 47-53

[17] World Medical Association. Declaration of Helsinki - Ethical principles for medical research involving human subjects. Available from http:// dl.med.or.jp/dl-med/wma/helsinki2013e.pdf Accessed March 11, 2016
[18] Stevens LA, Coresh J, Greene T et al. Assessing kidney function--measured and estimated glomerular filtration rate. N Engl J Med 2006; 354 : 2473-2483

[19] Guidance for Industry; Pharmacokinetics in Patients with Impaired Renal Function - Study Design, Data Analysis, and Impact on Dosing and Labeling. FDA, May 1998

[20] Note for Guidance on the Evaluation of the Pharmacokinetics of Medical Products in Patients with Impaired Renal Function. EMEA, June 2004

[21] National Kidney Foundation. K/DOQI Clinical Practice Guidelines for Chronic Kidney Disease: Evaluation, Classification and Stratification. Am J Kidney Dis 39: S1-S266 2002 (Suppl 1):

[22] Matsuo S, Imai E, Horio M et al. Revised equations for estimated GFR from serum creatinine in Japan. Am J Kidney Dis 2009; 53: 982-992

[23] Yale JF, Bakris G, Cariou B et al. Efficacy and safety of canagliflozin in subjects with type 2 diabetes and chronic kidney disease. Diabetes Obes Metab 2013; 15: 463-473

[24] Kohan DE, Fioretto P, Tang W et al. Long-term study of patients with type 2 diabetes and moderate renal impairment shows that dapagliflozin reduces weight and blood pressure but does not improve glycemic control. Kidney Int. 2014; 85: 962-971

[25] Barnett AH, Mithal A, Manassie J et al. Efficacy and safety of empagliflozin added to existing antidiabetes treatment in patients with type 2 diabetes and chronic kidney disease: A randomised, double-blind, placebo-controlled trial. Lancet Diabetes Endocrinol 2014; 2: 369-384

[26] Kashiwagi A, Takahashi $\mathrm{H}$, Ishikawa $\mathrm{H}$ et al. A randomized, doubleblind, placebo-controlled study on long-term efficacy and safety of ipragliflozin treatment in patients with type 2 diabetes mellitus and renal impairment: Results of the long-term ASP1941 safety evaluation in patients with type 2 diabetes with renal impairment (LANTERN) study. Diabetes Obes Metab 2015; 17: 152-160

[27] Haneda $M$, Seino $Y$, Inagaki $N$ et al. Influence of Renal Function on the 52-Week Efficacy and Safety of the Sodium Glucose Cotransporter 2 Inhibitor Luseogliflozin in Japanese Patients with Type 2 Diabetes Mellitus. Clinical Therapeutics 2016; 38: 66-88

[28] Wanner C, Inzucchi SE, Lachin JM et al. Empagliflozin and progression of kidney disease in type 2 diabetes. N Engl J Med 2016; 375: 323-334

[29] Mahaffey KW, Neal B, Perkovic V et al. Canagliflozin for Primary and Secondary Prevention of Cardiovascular Events: Results From the CANVAS Program (Canagliflozin Cardiovascular Assessment Study). Circulation. 2018; 137: 323-334

[30] Jerums G, Premaratne E, Panagiotopoulos S et al. The clinical significance of hyperfiltration in diabetes. Diabetologia. 2010; 53: 2093-2104

[31] Ruggenenti P, Porrini EL, Gaspari F et al. Glomerular hyperfiltration and renal disease progression in type 2 diabetes. Diabetes Care. 2012; 35: 2061-2068 